NASA-TM-112487

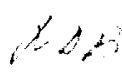

Pergamon

Applied Supercondurtatity Vol. 2. No. 78. pp. 479.486 .1994

Copyright i: 1995 Elsevier Science Lid

$0964-1807(94) 00040-9$

Printed in Great Britain. All rights reserved

$09641807 / 95 \$ 9.50+0.00$

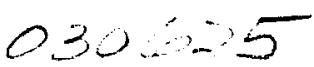

\title{
A NATURAL APPLICATION FOR HIGH TEMPERATURE SUPERCONDUCTORS: A BEARING FOR THE AZIMUTH MOUNT OF A LUNAR TELESCOPE
}

\author{
Ki Ma,' Mark Lamb, 'Peter Chen, ' Thomas Wilson, 3 Rodger Cooley, \\ Harold Xia, 'Clay Fowler,' Quark Chen' and Wei-Kan Chu' \\ 'Texas Center for Superconductivity and Department of Physics. University of Houston. \\ Houston. TX 77204-5932. \\ 'Computer Sciences Corporation. Calverton. MD 20705. 'Johnson Space Center. \\ National Aeronautics and Space Administration. Houston. TX 77058-3696, U.S.A
}

\begin{abstract}
A bearing for telescope mounts on the moon has to function in a cold dusty vacuum environment that impairs the operation of almost all traditional bearings, but it is a natural environment for bearings constructed out of magnets and high temperature superconductors. The challenge lies not so much in the weight of the telescope that has to be supporied. but in the smoothness of forces required for precision positioning control over a long stretch of time without human intervention. In this paper, we present a design of hybrid superconductor magnet bearings intended for use on the azimuth mount of an altitude-azimuth telescope mount system. In addition to the general features of hybrid super conducting magnet bearings, we will address particular issues connected with the application of these bearings on a telescope mount
\end{abstract}

\section{INTRODUCTION}

The moon has long been a coveted site for an observatory with the astronomers. It offers cold, dark vacuum conditions of interplanetary space combined with the stability of a massive anchor. Together with the 27-day rotation period of the moon, it allows the astronomer to have long continuous periods of observation under viewing conditions free from atmospheric disturbances and other sources of interference.

However, the moon has been quite inaccessible until this century. Even now, visits to the moon can only be infrequent. Moreover, the payload that can be carried there is limited to about $200 \mathrm{~kg}$. This implies that telescopes to be stationed on the moon must be extremely lightweight, and able to. function by itself for long periods of time (estimated at 5 years) without on site human maintenance. The mass of the telescope assembly and structure can be minimized by using materials and fabrication technology proven in space applications, to produce precision optical elements of very low areal density $\left(<2 \mathrm{~kg} / \mathrm{m}^{2}\right)$ [1].

While the lunar environment is ideal for observations, it is harsh on the equipment with at least two distinct components, the electronics involved in imaging and the mechanics of the bearings on the telescope mounts. The difficulty with the electronic component is being overcome with a new generation of ultraviolet detectors using charge-injection devices [2]. Here, we propose to utilize superconductor magnet bearings to circumvent difficulties associated with the mechanics of the mount.

Existing machinery that can achieve the kind of extremely fine motion required to track a star from the moon are massive, require carefully controlled environments, and need periodic maintenance, rendering them unsuitable for use on the moon. Moreover, the low temperature of the lunar surface requires the use of solid lubricants, which suffer from torque noise problems.

On the other hand, this low temperature is sufficiently low for the recently discovered high temperature ceramic superconductors (HTSs) to work with only minimal amount of thermal shielding, without the use of cryogens. One of the earliest suggestions for application of this novel material was in levitation magnet bearings [3-6]. Such superconductor magnet bearings would operate naturally in the cold vacuum environment on the moon if due care is taken to protect the telescope from the Sun's heat, either with heat shields or placed in a deep crater at the poles. These bearings exhibit multi-axis stability and they are passive (i.e. require no power consumption for operation. There is no mechanical contact between the rotor and stator of the bearings, and hence 
do not wear out with use and require no maintenance. For the same reason, they are essentially frictionless, permitting electronic positioning control with exceedingly fine resolution and an extended range of motion.

Besides being cold, the lunar surface is also constantly bombarded by particle radiation from solar flares and the solar wind. These charges up lunar dust particles, causing them to jump sometimes as high as $1 \mathrm{~m}$ across the terminator from the light to the dark side. Our bearing is tolerant of such dust storms, since there is a wide gap between the rotor and the stator. One might also be concerned with the effect of such particle radiation on the electromagnetic property of the HTS itself. The crucial electromagnetic property of the HTS material for holding the bearing together is its critical current density. As it turns out, the cumulative effects of 5 years of exposure on the lunar surface can be expected to improve the critical current density slightly $[7,8]$, and hence should not constitute an impediment to the performance of the bearing during this time period.

\section{HTS/MAGNET INTERACTION}

There has been a long history in the effort to exploit magnetic forces to create noncontact bearings. Magnetic bearings. invariably incorpqrate active control circuits because a system consisting of permanent magnets and/or ferromagnetic or paramagment materials is inherently unstable, a result embodied in Earnshaw's theorem [9]. Indeed, the active magnetic bearing is a highly developed and sophisticated device.

Stable configurations become possible if we introduce diamagnetic materials. A case in point is that of a permanent bar magnet, levitated above a hemispherici: bowl of lead at liquid helium temperatures [10]. Here, lead, being a type I superconductor. 'h haves as a perfect diamagnet. Unlike lead, the HTSs are type II superconductors. Their behavior is more akin to that of an almost perfect conductor than that of a perfect diamagnet. A perfect conductor has a negative susceptibility towards differential increments in a magnetic field. just as a perfect diamagnet. However, any magnetic field already present would not be expelled. but would remain trapped in a perfect conductor. The implication for magneto-mechanics is that the levitation force on a magnet placed on a piece of HTS material which is then cooled is small, but the stiffness, which stabilizes the position of the magnet, remains appreciable.

\section{HYBRID SUPERCONDUCTOR MAGNET BEARING}

The above prediction has been demonstrated by actual experiment. We can construct a magnetic bearing in which active control is replaced by the stabilizing action from a HTS. The HTS is cooled after being assembled into the bearing, and is neither expected nor required to provide any thrust. This is the essential concept of the hybrid superconductor magnet bearing $[4,5]$

To function as a bearing, a rotor must rotate freely about an axis with respect to a stator. It is important to note that, although the magnetic flux penetrating the volume of the HTS is strongly pinned. this force is not transmitted outside the HTS. and in particular. not to the permanent magnet although they are linked via the magnet flux lines. The analogy with a conductor suggests that it is a change in the magnetic field that the HTS responds to. If the magnets on the rotor are azimuthally symmetric about the rotation axis, the rotational motion of the rotor magnets do not incur any change of magnetic field in the HTS. Hence. there will be no drag force against the rotation of the rotor magnets. This has also been directly verified by experiments [11].

In practice. it is often found that the stabilization that the HTS can provide is not quite sufficient to overcome the instability of the magnets. We have employed two approaches to make up for this. One approach enhances the stability with high gradients of the magnetic field "frozen" into the HTS. This is easy to understand as the converse of the argument in the last paragraph. A higher gradient of the magnetic field results in a larger change in the magnetic lield in the superconductor for a given displacement that produces a stronger restoring force.

The other attempts to stabilize the magnet system itself. This can be achieved most readily by using symmetry to balance the instabilities off each other. For a system of rotor and stator magnets 
with cylindrical symmetry and opposite parity (i.c. if $\mathbf{B}(-\mathbf{r})=\mathbf{B}(\mathbf{r})$ for the rotor magnet system, then $\mathbf{B}(-\mathbf{r})=-\mathbf{B}(\mathbf{r})$ for the stator magnet system . It can be shown that the stiffnesses in all the translational and rotational directions vanish. but that a finite thrust remains on the rotor from the stator. With further refinement, we can proceed one step further to eliminate cross coupling between translation in a direction perpendicular to the symmetry axis and rotation about the axis perpendicular to both. This creates a system with a neutral equilibrium that is, in a certain sense, the most stable system that can be achieved without violating Earnshaw's theorem. In practice, a small instability always remains. due to imperfections. These should be small enough to be rendered insignificant by the action of the HTS.

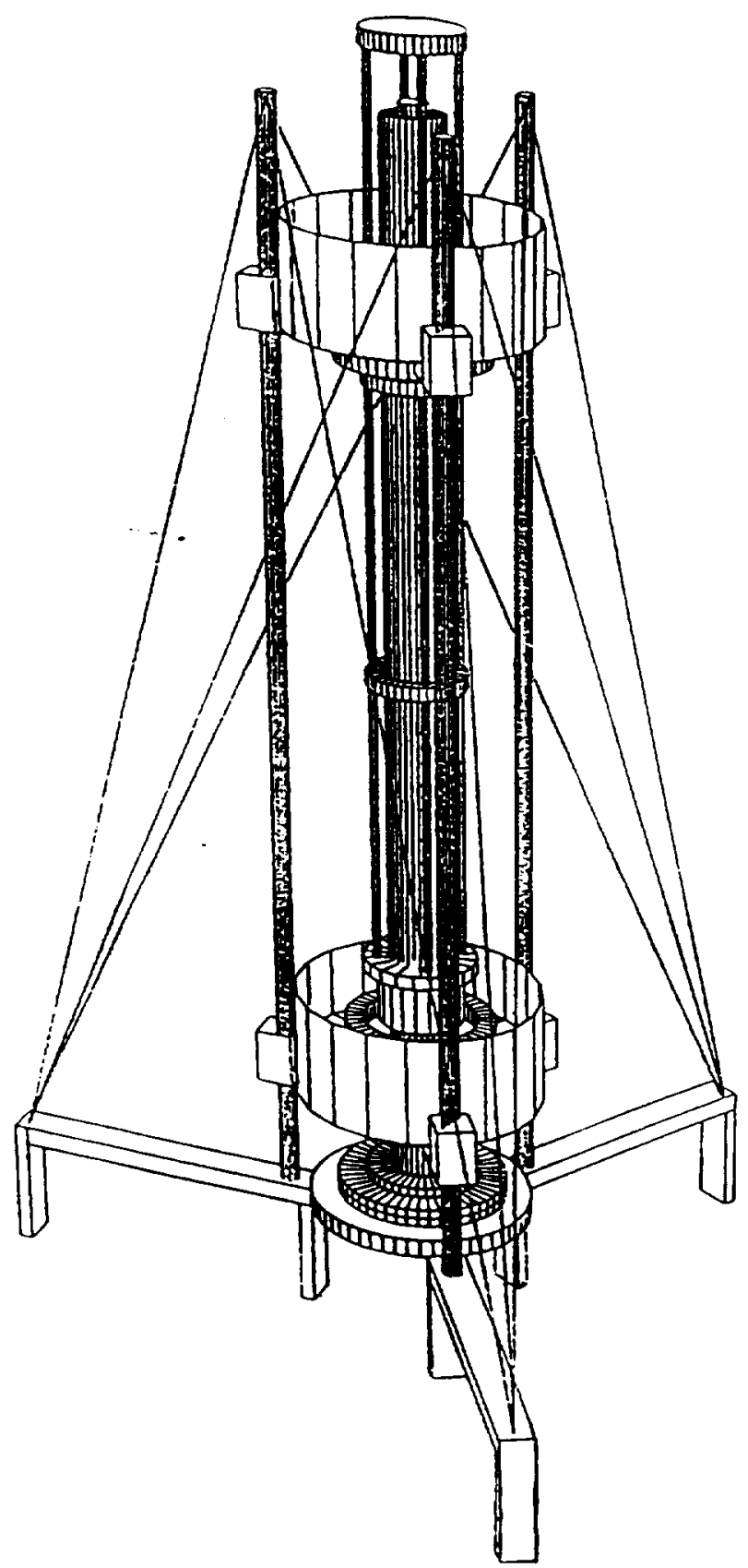

Fig. 1. Three-dimensional overview of bearing assembly 


\section{BEARING FOR LUNAR TELESCOPE}

The bearing on the azimuth mount of a lightweight telescope on the moon need support only a very light load, but stability is still essential. On earth, this is achieved by using counterweights, so that the center of gravity of the telescope assembly falls below the pivotal point of support. This entails additional mass to be added to the telescope assembly, which is undesirable for a telescope to be transported to the moon. Our bearing desien takes care of this problem by providing an effective counterweight with magnetic forces in t:a same way that these were utilized to support the weight of the telescope assembly itself. A three-dimensional overview of the bearing is presented in Fig. 1. A cross-sectional view is shown in Fig. 2 and a schematic of the magnets and HTSs used in the bearing is displayed in Fig. 3.

As can be visualized from these figures, our bearing design consists of a pair of bearings with opposite thrusts. The bottom bearing provides a downward thrust, and acts as the counterweight. The top bearing provides the thrust that is the sum of this counterweight and the actual weight of

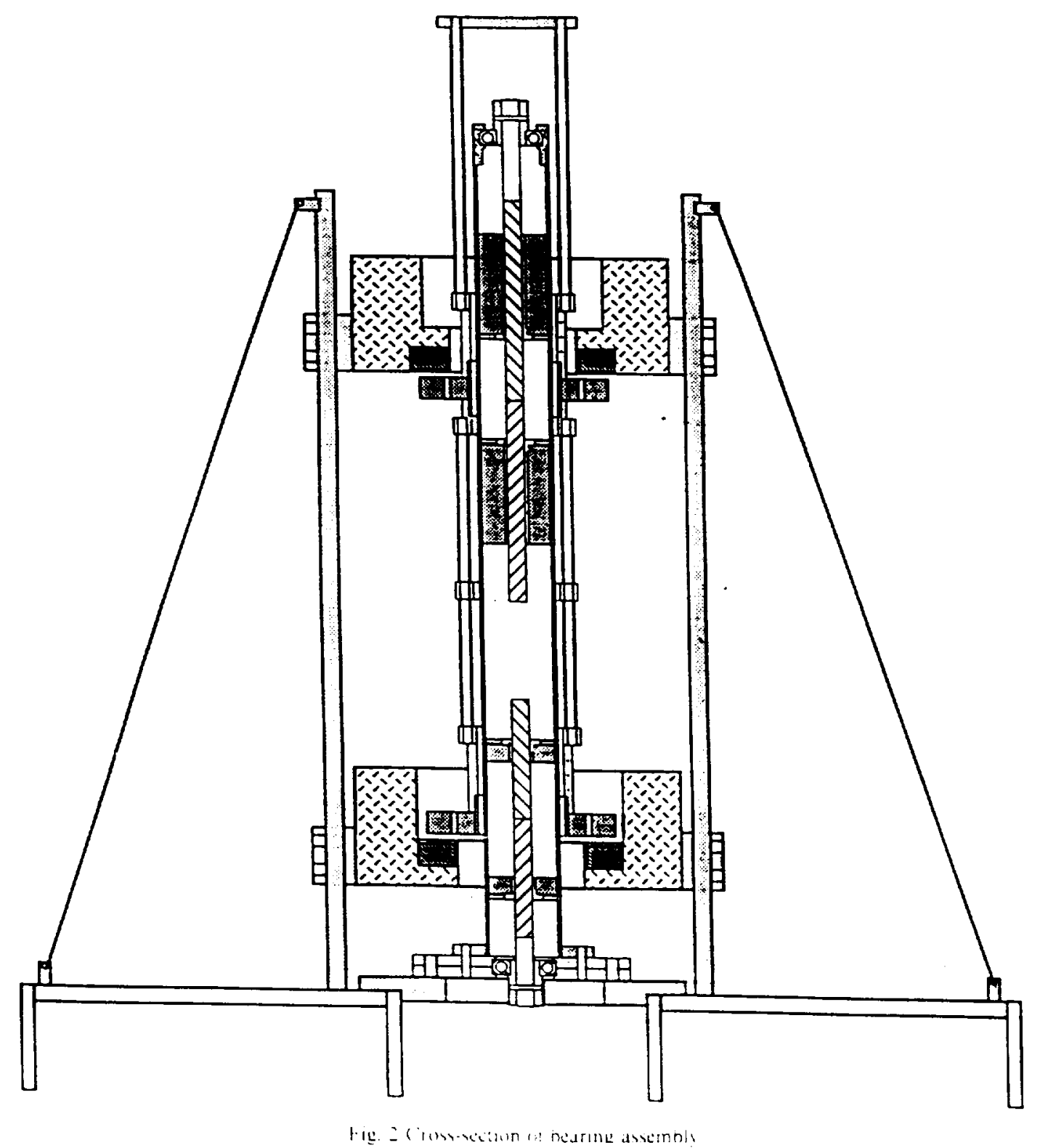




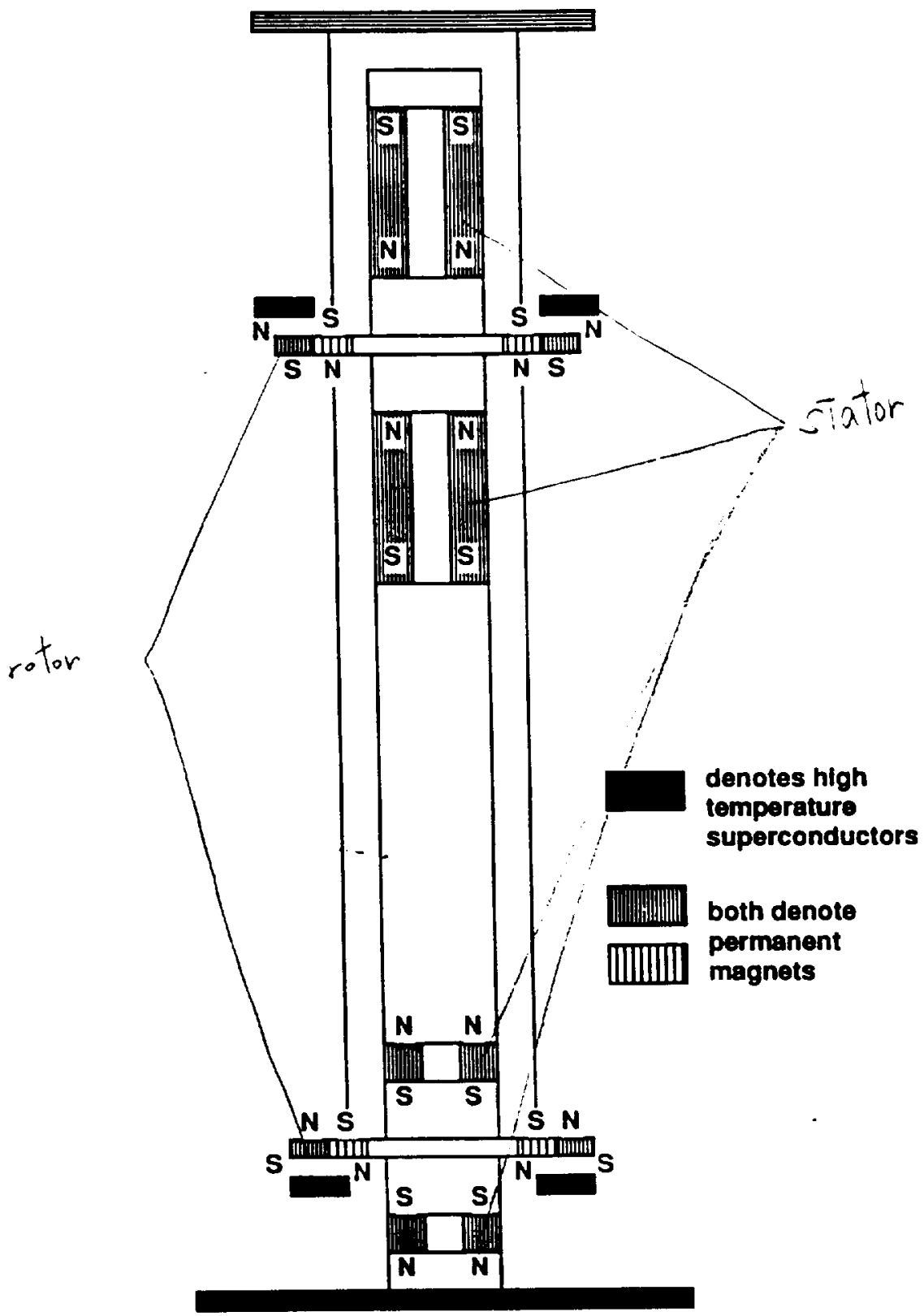

Fig. 3. Schematic diagram showing the magnets and superconductor comprising the bearings.

the telescope assembly. Each of these bearings is designed with neutral stability. The rotor magnet consists of two concentric ring magnets, one fitted into the other with opposite polarity. The magnets were designed to strengthen the stability obtainable from the HTSs by maximizing the gradient of the magnetic field that would be frozen into the superconductors. However, the opposite polarity arrangement also implies that the thrust that can be obtained from this volume of magnetic material is just the net of two opposing thrusts, and is not the optimal. We can afford to do this here, as the weight to be supported is not excessive. The stator magnets are pairs of cylindrical magnets with a center hole, placed symmetrically above and below the corresponding rotor magnets. The stator magnets of the top bearing is taller to provide a higher thrust. The distance between the stator magnets in each of these pairs can be adjusted without destroying the symmetry of the positioning with respect to the corresponding rotor magnet. This allows the thrusts provided by these bearings to cover a finite range without giving up the condition of neutral stability for the magnets. 

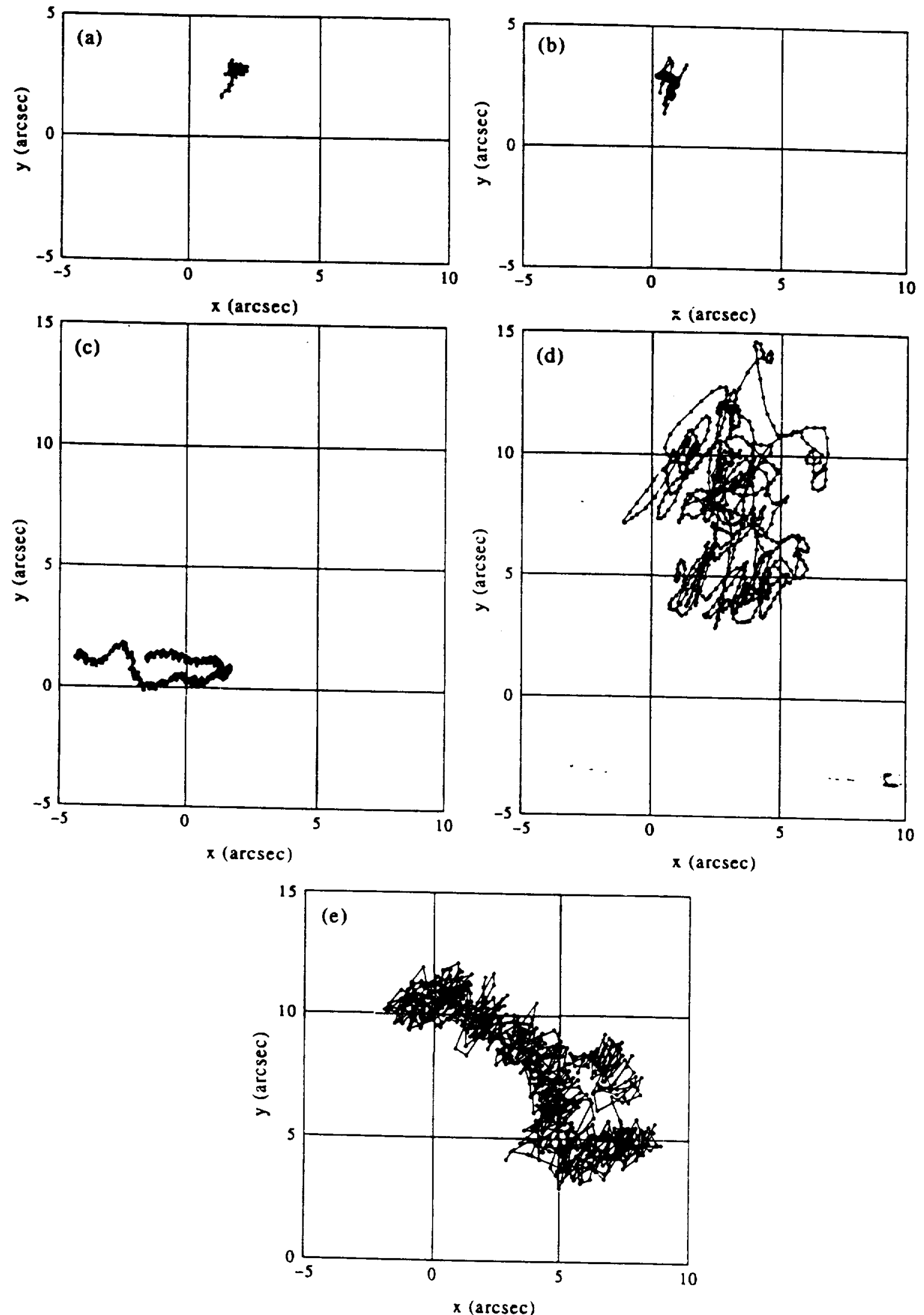

Fig. 4. Traces of laser spot allgular positions for observation periods of $(a) 8 \mathrm{~ms}$. (b) $70 \mathrm{~ms}$. (c) $0.5 \mathrm{~s}$. Id 8 s and (e) 32 s. respectuvely. 
PERFORMANCE OF BEARING

The top bearing can support an axial load of $45 \mathrm{~N}$. It has been used to support a model telescope with a weight of $12 \mathrm{~N}$, the equivalent counterweight of $12 \mathrm{~N}$ from the bottom bearing, and the weight of the levitated structure itself making up the remainder. The entire assembly has been observed to rotate continuously under its own inertia for over $45 \mathrm{~min}$. The bearing assembly excluding the telescope is about $1 \mathrm{~m}$ tall. The top plate is $11.4 \mathrm{~cm}$ in diameter, while the base spans $56 \mathrm{~cm}$. The central shaft is slightly less than $7.6 \mathrm{~cm}$ in diameter. The levitated structure is held in place with a total stiffness from the bearings of about $10 \mathrm{~N} / \mathrm{mm}$ in the axial direction. This low stiffness will be supplanted by the stiffness due to feedback control in the complete system. The HTSs are enclosed in two structures $7.6 \mathrm{~cm}$ tall and $16.5 \mathrm{~cm}$ in diameter, made of Styrofoam and G-10 glass fibre/epoxy composite and fixed separately at 25 and $84 \mathrm{~cm}$ from the top. With all the necessary fixtures included. the whole bearing assembly weighs $67 \mathrm{~N}$

To the astronomer, an important performance parameter is the stability of the bearing. In order to determine this, we mounted a mirror on the bearing and directed a laser beam to be reflected from the mirror onto an $x-y$ position sensor. We monitored the position of the beam on the $x-y$ position sensor for continuous time periods of different lengths, from $8 \mathrm{~ms}$ to $32 \mathrm{~s}$. Traces of the laser spot on the sensor are reproduced in Fig. 4. The accumulated spot size, defined by the standard deviation of the angular position from the average over the entire observation period, is plotted as a function of the observation period in Fig. 5. We can see that the spot size increases from about $0.97 \mu \mathrm{rad}$ of arc at $8 \mathrm{ins}$ of time to around $19.4 \mu \mathrm{rad}$ of arc after $32 \mathrm{~s}$ of time. For observations from the moon, an accuracy of pointing to $0.48 \mu \mathrm{rad}$ of arc is desirable. The angular measures were converted from the position on the $x-y$ position sensor by dividing over the distance $(3 \mathrm{~m})$ from the mirror to the $x-y$ position sensor.

Before a telescope is fully functional, other parts, most notably the drive mechanism itself and Tts associated control, must be developed, and then integrated into the system. What we have shown is that this control motor mechanism that is eventually responsible for tracking stars would have to be able to act within a time frame shorter than $8 \mathrm{~ms}$. The bearing itself can use a shorter height. Horizontal and tilted versions await development, as these would be needed in the altitude or equatorial mounts and lifetime and reliability tests must be performed.

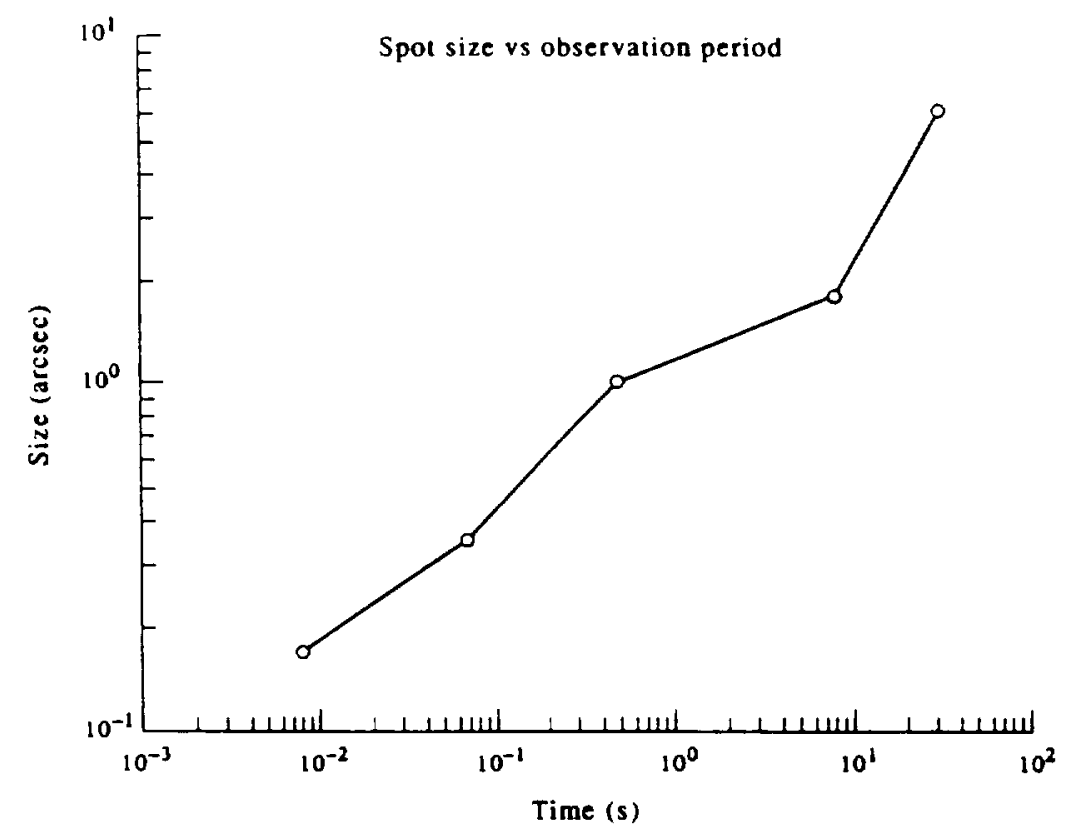

Fig. 5. Spot size (as measured by the standard deviation from mean positioni as a function of observation period. 


\section{SUMMARY}

We have demonstrated that the hybrid superconductor magnet bearing holds promise as a viable solution to the problem of fabricating a bearing for the exceedingly fine drive mechanism required for a lunar telescope.

Although developed for use on the moon, these bearings could conceivably be applied on earth as well, provided that a cold vacuum environment is created for their accommodation. In some applications, such as cryogenic turbopumps, such an environment may already exist for other reasons. In others, such as flywheels for kinetic energy storage [11], the inconvenience of having to maintain a cold vacuum might be acceptable as a small price for a large gain in performance. We hope that these bearings will find themselves useful and be of service in numerous applications, on the moun, in space as well as on earth.

Acknowledgements-This work is supported in part by the Advanced Research Project Agency and in part by the State of Texas through the Texas Center of Superconductivity at the University of Houston. The collaboration and hospitality of R. J. Oliversen at NASA/Goddard Space Flight Center, H. Hojaji at Catholic University of America and R. E. Pitts at Computer Sciences Corporation is gratefully acknowledged. We are also indebted to excellent suggestions from Chase McMichael at the University of Houston.

\section{REFERENCES}

I. P. C. Chen. R. E. Pitts, R. J. Oliversen. J. D. Stolarik. K. Segal, T. L. Wilson, E. I. Lin, J. R. Hull, R. Romeo, H. Hojaji, K. B. Ma. Q. Y. Chen, W. K. Chu and C. W. Chu, Bull. Am. Astron. Soc. 25, 1303 (1993).

2. L. J. Payne, R. A. Kimble, A. M. Smith, J. P. Haas, C. C. Sturgell Jr. R. E. Wentink, J. Carbone and P. C. Chen. Bull. Am. Astron. Soc. 25, 1304 (1993).

3. F. C. Moon. P. Z. Chang, Appl. Phys. Lett. 56, 397 (1900).

4. C. K. McMichael, K. B. Ma. M. W. Lin, M. A. Lamb, R.-L. Meng, Y. Y. Xue, P. H. Hor and W. K. Chu, Appl. Phys. Lett. 59, 2442 (1991)

5. W. K. Chu, K. B. Ma and C. K. McMichael. US Patent No. 5,159,219. October 27 (1992).

6. M. Komori. T. Matshushita and M. Takeo. Cryogenics 33, 1058 (1993).

7. Y. J. Zhao. W. K. Chu, M. F. Davis, J. C. Wolfe. S. C. Deshmukh. D. J. Economou and Anne McGuire, Physica $C$ 184. $144(1991)$.

8. W. K. Chu. J. R. Liu and Z. H. Zhang, Proc. Th Int. Conf. on Ion Beam Modification of Materials, Knoxville (September 1990).

9. S. Earnshaw, Trans. Camb. Phil. Soc. 7, 97 (1842).

10. K. Mendeissohn. The Quest for Absolute Zero. Tayior \& Francis, London (1977)

11. Q. Y. Chen. K. B. Ma. C. K. McMichael, M. A. Lamb. R. S. Cooley, P. C. Fowler. H. Z. Xia. R.-L. Meng. C. W. Chu and W K. Chu. Proc sith US Japan Workshop on High Temperature Superconductors. Houston (December 1993) 